\title{
Tomographic Imaging of BiologicalTissue by Time-Resolved, Model-Based, Iterative Image Reconstruction
}

\author{
Andreas H. Hielscher ${ }^{1}$, Alexander Klose ${ }^{1,2}$, David Catarious J. ${ }^{1}$, and Kenneth M. Hanson ${ }^{2}$ \\ Los Alamos National Laboratory \\ ${ }^{1}$ Bioscience and Biotechnology, CST-4 \\ ${ }^{2}$ Image Analysis, DX-3 \\ MS E535, Los Alamos, New Mexico 87545, \\ Tel. 505667 7270, Fax 505665 4637, E-mail: hielsch@lanl.gov
}

\begin{abstract}
Currently available tomographic image reconstruction schemes for photon migration tomography (PMT) are mostly based on the limiting assumptions of small perturbations and a priori knowledge of the optical properties of a reference medium. In this work a modelbased iterative image reconstruction (MOBIIR) method is presented, which does not require the knowledge of a reference medium or that the encountered heterogeneities are small perturbations. After a description of the major code structure and a review of the mathematical background, the clinically relevant examples of brain imaging and breast cancer detection and are discussed. It is shown that ventricular bleedings in the brain can be detected and that cysts and tumors in the breast can be distinguished using the MOBIIR technique.
\end{abstract}

\section{Keywords}

100.6950 Tomographic image processing; (170.3010) Image reconstruction techniques; (170.3880) Medical and biological imaging; (170.6920) Time-resolved imaging; (290.7050) Turbid media

\section{Introduction}

In recent years researchers have invested considerable efforts towards tomographic imaging systems that use nearinfrared (NIR) light. ${ }^{1,2,3,4}$, Besides Photon Migration Tomography (PMT) various other names are commonly used for this novel imaging modality, such as Medical Optical Tomography, Diffusion Tomography, or Transillumination Imaging, to name a few. Based on measurements of transmitted and/or reflected light intensities on the surface of the medium, a reconstruction is attempted that provides the spatial distribution of the optical properties, e.g. absorption coefficient, $\mu_{\mathrm{a}}(\mathbf{r})$, and scattering coefficient, $\mu_{\mathrm{s}}(\mathbf{r})$, inside the medium. In initial clinical trials, performed by various groups around the world, PMT has shown great promise for brain-bloodoxygenation monitoring in preterm infants, hematoma detection and location, cognition analysis, and breast cancer diagnostics. ${ }^{5}, \frac{6}{6}, 8,9,9$

The technology for making light-transmission measurement on human subjects is nowadays readily available $.5,6,{ }^{10},{ }^{11},{ }^{12}$ However, a major challenge remains the development of computer algorithms that transform these measurements into useful images of the interior of large organs. Other than x-rays, the near-infrared photons used in PMT do not cross the medium on a straight line from the source to the detector. Light is scattered and absorbed throughout the system. Hence, standard backprojection method have only limited success $,{ }^{13},{ }^{14}$ and other analytical reconstruction methods are only available for highly restrictive problems. ${ }^{15},{ }^{16,17}$ In general, either the optical properties of a reference medium have to be known, or/and it has to be assumed that the heterogeneities constitute only a small perturbation in a homogeneous background medium. To overcome this limitation research has increasingly turned to model-based iterative reconstruction (MOBIIR) algorithm. ${ }^{18,19,20,21}$

The goal of these algorithms is to reconstruct an image of the optical properties (absorption, $\mu_{\mathrm{a}}(\mathbf{r})$, and scattering, $\mu_{\mathrm{s}}(\mathbf{r})$, coefficients; $\mathbf{r}$ is spatial location) inside a medium, from a given set $\mathbf{M}$ of measurements on the circumference of the medium. These iterative reconstruction schemes in general consist of three components (see Fig. 1): (1) Forward Model: This model is a theory or algorithm that 
predicts a set of measured signals, $\mathbf{P}$, based on the position of the light source and the spatial distribution, $\zeta=\left(\mu_{\mathrm{a}}(\mathbf{r})\right.$, $\mu_{\mathrm{S}}(\mathbf{r})$ ), of optical properties; (2) Analysis Scheme: Here an objective function, $\phi$, is defined, which describes the difference between the measured and predicted data. A simple example is the least-square error norm $\phi(\zeta) \sim(\mathbf{M}-\mathbf{P}(\zeta))^{2}$;. Since the problem is highly ill-posed, a regularization term, $\mathrm{R}$, is usually added to the objective functions. (3) Updating Scheme: Once the objective function is defined, the task becomes to minimize $\phi$ by successively modifying the distribution $\zeta$. This means that the parameters of the forward model have to be update to generate a second, third, etc., prediction $\mathbf{P}$, which can be compared to the actual measurement data $\mathbf{M}$. The reconstruction is complete once a distribution $\xi$ is found for which $\phi(\zeta)$ is smallest.

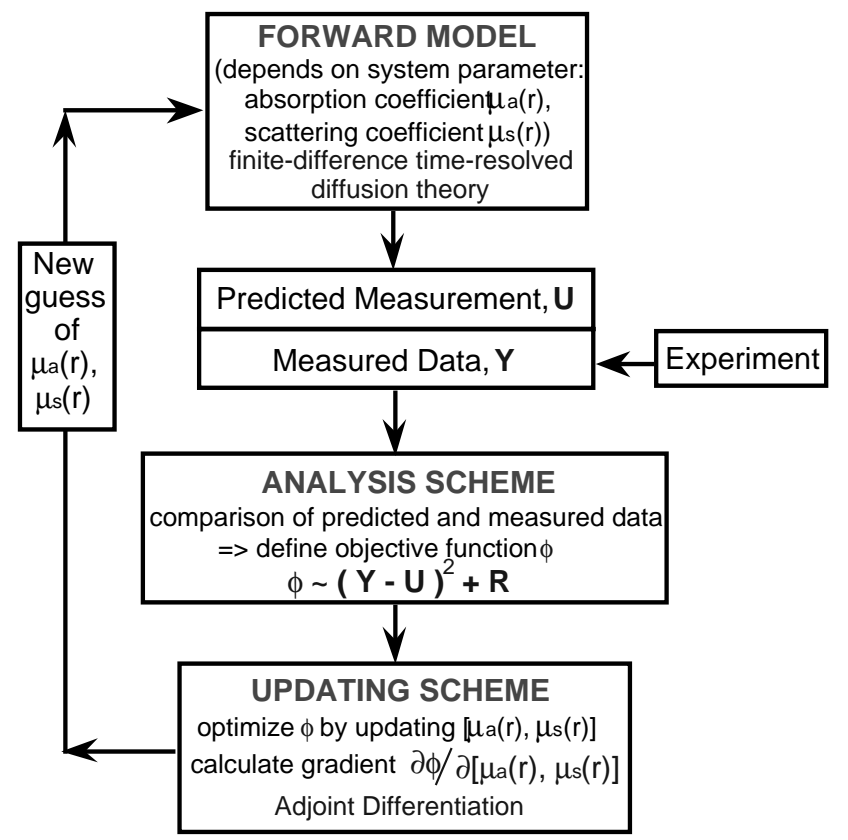

Fig. 1. Flow diagram of model-based iterative image reconstruction scheme.

In this work I will first review some details of the MOBIIR code, which was recently developed at Los Alamos National Laboratory. ${ }^{20}$ The three components, (1) forward model, (2) analysis scheme, and (3) updating scheme, used in this particular algorithms are outlined and discussed. This is followed by several simulated reconstruction examples, which illustrate the performance of this code. Besides simple reconstructions of well defined objects in a homogeneous background, reconstructions of breast and brain tissue are presented.

\section{Mathematical Background}

\subsection{Forward Model}

As a forward model that describes the photon propagation in the turbid medium and predicts the measurements at the detector position $\mathbf{r}_{\mathbf{d}}$, we use in this study a 2-dimensional, time-resolved diffusion equation:

$$
\frac{\partial U}{\partial t}=\frac{\partial}{\partial x_{p}}\left(D \frac{\partial U}{\partial x_{p}}\right)-c \mu_{a} U+S, \quad p \in\{1,2,3\}
$$

where $U(x, y, t)$ is the diffuse intensity and $S(x, y, t)$ is the source strength at position $\left(x_{1}, x_{2}, x_{3}\right)$ and time $t$.The position-dependent absorption and diffusion coefficients

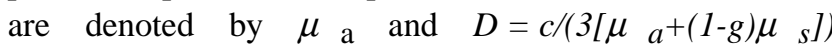
respectively. Here $\mathrm{c}$ is the speed of light in the medium and $\mathrm{g}$ is the scattering anisotropy value, equal to the average value of the cosine of the angle through which photons are scattered. $\mu_{s}{ }^{\prime}=(1-g) \mu_{s}$ is known as the effective or transport scattering coefficient.

The equation (1) is solved by replacing the temporal and spatial derivatives by their finite-difference approximations as follows:

$$
\begin{aligned}
& \frac{\partial U}{\bar{\partial} t} \approx \frac{U^{n}-U^{n-1}}{\Delta t} \\
& \frac{\partial}{\partial x_{p}}\left(D \frac{\partial U}{\partial x_{p}}\right) \approx \delta_{x_{p}}\left(U_{i, j, k}, D_{i, j, k}\right)
\end{aligned}
$$

By substituting the finite-difference approximations $(3,4)$ in the diffusion equation (1), we obtain a difference equation that needs to be solved forward in time. The finite-difference approximations to the spatial derivatives (4) can be evaluated at time index $n+1$ or $n$, when we are solving the difference equation for $U^{n+1}$. Methods that evaluate the spatial derivatives (4) at the past time instance (n) are called explicit, while methods that evaluate the spatial derivatives (4) at the present time instance $(n+1)$ are called implicit. Here we use a technique known as "alternating directions implicit" (ADI) method. In this method, the computation of $\mathrm{U}^{\mathrm{n}+1}$ from $\mathrm{U}^{\mathrm{n}}$ is broken up in three time steps. In the first 1/3 time step, only the spatial derivative in one direction is evaluated at the present time instance (implicit) and the other spatial derivatives are evaluated at the previous time instance (explicit). In the next $1 / 3$ time step, the implicit and explicit directions are switched, etc..

We can compactly write the ADI method as

$$
\begin{aligned}
A U^{n+1 / 3} & =(B+C) U^{n}+S^{n+1 / 4} \\
\text { or } \quad \quad \quad U^{n+1 / 3} & =A^{-1}(B+C) U^{n}+A^{-1} S^{n+1 / 4}
\end{aligned}
$$

The structures of the matrices $\mathrm{A}$ and $\mathrm{D}=(\mathrm{B}+\mathrm{C})$ remain the same in all 3 1/3-time steps but the absolute values of the matrix elements differ. The advantage of the ADI method is that by correct ordering the matrix A can always be made triadiagonal, e.g., for the first 1/3 step:

$$
A_{i i}=1+c \mu_{a} \frac{\Delta t}{3}+\left(D_{i+1 / 2, j, k}+D_{i-1 / 2, j, k}\right) \frac{\Delta t}{3 \Delta x}
$$




$$
A_{i, i \pm 1}=-D_{i \pm 1 / 2, j, k} \frac{\Delta t}{3 \Delta x}
$$

The matrix A needs to be inverted to compute $U^{n+1 / 3}$ from $U^{n}$ (Eq. 6b). Since A is always tri-diagonal the inversion can be easily done in $0(\mathrm{~N})$ computation. Furthermore, the ADI method is unconditionally stable for any value of $\Delta \mathrm{t}$. From now on, we do not distinguish between the three 1/3-time stesp for notational simplicity. For further information on explicit, implicit and ADI methods see and Press et al. ${ }^{22,},{ }^{23},{ }^{24}$ ?

\subsection{Analysis Scheme}

The forward model is used to calculate detector responses at a set of detector position $M$. Let $Y$ denote the measurements of the diffuse intensity $U$ for all $s \quad M$. We will assume in this paper for simplicity that the measurements are corrupted by uncorrelated Gaussian noise. However, note that the method we propose is not limited to this choice, and we could as well have chosen a more complex model such as poisson noise. In this work the objective function is defined as:

$$
\Phi(\zeta)=\sum_{s \in M} 1 / 2 \sigma(s)^{2} \sum_{n}\left(Y_{s}^{n}-U_{s}^{n}(\zeta)\right)^{2}+R(\zeta)
$$

Here $\mathrm{R}(\zeta)$ describes any additional regularization term, which in general depends on the spatial distribution of optical properties $\zeta=\left[\mathrm{c}_{\mathrm{a}}(\mathrm{r}), \mathrm{D}(\mathrm{r})\right]$. For example, assume that we know the spatial distribution of optical properties, $\zeta_{\mathrm{H}}$, of a healthy breast without any tumors or cysts. We could use this information for the reconstruction by defining

$$
R(\zeta)=\lambda \sum_{r}\left|\frac{\zeta_{H}(r)-\zeta(r)}{\sigma(r)}\right|^{p}
$$

The parameter $\lambda$ is a weighting factor for the regularization term, which determines the influence of the regularizer on the final solution. The regularization term compares $\zeta$ at position $\mathbf{r}$, with $\zeta_{\mathrm{H}}$ at position $\mathbf{r}$. Differences between $\zeta_{\mathrm{H}}$ and $\zeta$ are more or less penalized depending on the choice of $\mathrm{p}$. In this work we used $\mathrm{p}=2.0$. Note that the noise variance $\sigma(s)$ is space varying.

The goal of the reconstruction algorithm is now to minimize this objective function, $\Phi$, by appropriately choosing the system parameters $D(\boldsymbol{r})$ and $c \mu{ }_{a}(\boldsymbol{r})$.

\subsection{Gradient Calculation}

The effective solution to optimization problems involving many variables (here the spatial distribution of optical properties) relies on knowing the gradient of the objective function with respect to the variable, $d \phi / d \zeta$. In this work we employ the method of reverse differentiation.. To outline this method we assume for simplicity that no regularization term is used, i.e. $R(\zeta)=0$. Furthermore we chose the following notation: $p, q, r, d \Omega$ are grid points; $d \Omega d \subset \Omega$ are detector positions on the boundary $\bar{\partial} \Omega$ and $\mathrm{M}$ denote measurments on the boundary $\bar{\partial} \Omega$. The derivative of the objective function (Eq. 8) with respect to the optical properties $\zeta$ is given by:

$$
\frac{d \Phi}{d \zeta_{r}}=\sum_{n \in T} \sum_{p \in \Omega} \frac{d \Phi}{d U_{p}^{n}} \frac{\partial U_{p}^{n}}{\partial \zeta_{r}}
$$

Here we simply applied the chain rule, and the first term in the sum is the outer derivative, while the second term is the inner derivative of Eq. 8. The second term is easily obtained by differentiating Eq. $6 a$ at time $n+1 / 3$ with respect to the optical parameters, which yields:

$$
\begin{aligned}
& \frac{d A}{d \zeta_{r}} U^{n+1 / 3}+A \frac{\partial U^{n+1 / 3}}{\partial \zeta_{r}}=\frac{d B}{d \zeta_{r}} U^{n}+\frac{d C}{d \zeta_{r}} U^{n} \\
\Rightarrow & \frac{\partial U^{n+1 / 3}}{\partial \zeta_{r}}=A^{-1}\left(\frac{d B}{d \zeta_{r}} U^{n}+\frac{d C}{d \zeta_{r}} U^{n}-\frac{d A}{d \zeta_{r}} U^{n+1 / 3}\right)
\end{aligned}
$$

Equation 11 can be easily calculated by means of the known intensity $\mathrm{U}$ and the matricies $\mathrm{A}, \mathrm{B}$ and $\mathrm{C}$ of the forward model.

More involved is the calculation of derivative of the objectiove function $\Phi$ with respect to the intensities $U^{n}$, which is the first term in Eq. (10). We obtain this term recursively by applying the chain rule and stepping backward (reverse) in time from $n+1 / 3$ to $n$,

$$
\frac{d \Phi}{d U_{p}^{n}}=\sum_{q \in \Omega} \frac{d \Phi}{d U_{q}^{n+1 / 3}} \frac{d U_{q}^{n+1 / 3}}{d U_{p}^{n}}+\frac{\partial \Phi}{\partial U_{p}^{n}} .
$$

with

$$
\text { (9) } \frac{d \Phi}{d U_{q}^{N}}=\frac{\bar{\partial} \Phi}{\partial U_{q}^{N}} \text { for } N=\max (n \in T) \text {. }
$$

Here, $\bar{o} \Phi / \bar{o} U_{p}^{n}$ denotes the change in $\phi$ when only $U_{p}{ }^{n}$ is varied keeping all other variables constant, while $d \phi / d U_{p}{ }^{n}$ denotes the total change in $\phi$ when $U_{p}{ }^{n}$ is varied along with all variables that depend on $U_{p}{ }^{n}$. Partially differentiating Eq. 8 with respect to $U_{p}{ }^{n}$, we obtain

$$
\frac{\partial \Phi}{\partial U_{p}^{n}}=\left\{\begin{array}{lr}
\frac{1}{\sigma^{2}}\left(M_{p}^{n}-U_{p}^{n}\right) & p=d \in \Omega_{d} \\
0 & \text { otherwise }
\end{array}\right.
$$

The term $\mathrm{dU}_{\mathrm{p}}{ }^{\mathrm{n}+1 / 3} / \mathrm{dU}_{\mathrm{p}}{ }^{\mathrm{n}}$ can again be calculated from the forward finite difference Eq. $6 \mathrm{~b}$ and we obtain:

$$
\frac{d U_{q}^{n+1 / 3}}{d U_{p}^{n}}=A^{-1}(B+C) I_{p} \text { with } I_{p}= \begin{cases}1 & q=p \\ 0 & q \neq p\end{cases}
$$


where $I_{p}$ is a column vector that is zero everywhere except at the spatial point $\mathrm{p}$, where it is unity.

The method of adjoint differentiation provides an efficient means to calculate the needed gradients for a complex sequence of calculations, such as the forward simulation of the migration of photons described above. Its power lies in its ability to calculate the derivatives with respect to all the variables in a CPU time that is comparable to the forward calculation.

\section{Results}

\subsection{Problem Setup}

The MOBIIR algorithm was tested by simulating timeresolved measurements on heterogeneous media and using this simulated measurements as input for the reconstruction code. The measurements were simulated with the finitedifference, time-resolved diffusion code. To mimic realworld measurements, gaussian noise was added to each measurement at a signal to noise (SNR) level of $30 \mathrm{db}$. This is a typical value for time-resolved single photon counting measurement ${ }^{25,26}$. When single photon counting is used, smooth data can be obtained over 2-3 orders of magnitude. Signals that are approximately 1000 times smaller than the maximum are increasingly effected by statistical noise.

Once the detector readings are simulated, they are used as the actual measurement data, $\mathrm{Y}$, in the reconstruction code. To start the reconstruction program an initial guess of the optical properties is necessary. If not stated otherwise a medium with a constant diffusion coefficient was used as a first guess. Based on this guess the forward code calculates the detector responses, $\mathrm{U}$, and compares these predicted measurements with the measurement data $\mathrm{Y}$, by calculating the objective function, $\phi$ (Eq. 7). The derivatives of $\phi$ with respect to $\zeta$ at all grid points is calculated and a new spatial distribution of $D$ and $\chi \mu a$ is put in place for the next forward calculation is . All calculations were done on a SUN Sparc10 workstation.

\subsection{Simple Systems}

As a first example a simple heterogeneous system is considered (Fig. 2a), which consists of a background medium with $\mathrm{D}=1.5 \mathrm{~cm}^{2} \mathrm{~ns}^{-1}$ and three objects with $\mathrm{D}=0.73 \mathrm{~cm}^{2} \mathrm{~ns}^{-1}$ and $6.7 \mathrm{~cm}^{2} \mathrm{~ns}^{-1}$ respectively. To simulate an $8 \times 8 \mathrm{~cm}$ medium a $40 \times 40$ x-y grid with a spatial resolution of $0.2 \mathrm{~cm}$ is used. The medium is surrounded by 16 source detector position. Moving the source around the medium results in $16 \times 15=240$ detector readings. Each detector reading consists of 50 time points, which are equally space by $\Delta t=0.1 \mathrm{~ns}$. In the reconstruction code the derivatives of each time-point with respect to each grid point is calculated, which results in $(240 \times 50) \times(40 \times 40)=$ $12000 \times 1600=19.200 .000$ derivative calculations in each iteration step. Fig $2 \mathrm{~b}$. shows the reconstructed D-image, which was obtained after 15 iteration (20 minutes). The initial guess was a homogeneous medium with $\mathrm{D}=1.2$ $\mathrm{cm}^{2} \mathrm{~ns}^{-1}$. The locations of the three objects are reconstructed with high accuracy. The code is also able to identify if the heterogeneity has a higher or lower diffusion coefficient than the background. However, the absolute values of $\mathrm{D}$ are not as accurate. This has been observed before by other researchers using different diffusion based image algorithms.

Fig. 2c shows the reconstruction of the absorption coefficient. Again the location of the absorbers is determined with high accuracy, however the absolute values are to small. Furthermore, some "cross talk" between the $\mathrm{D}$ and the $c \mu_{\mathrm{a}}$ reconstruction can be observed. Strong scattering inhomogeneities are misinterpreted as having a scattering and absorption component. The reason for this is not yet fully understand.

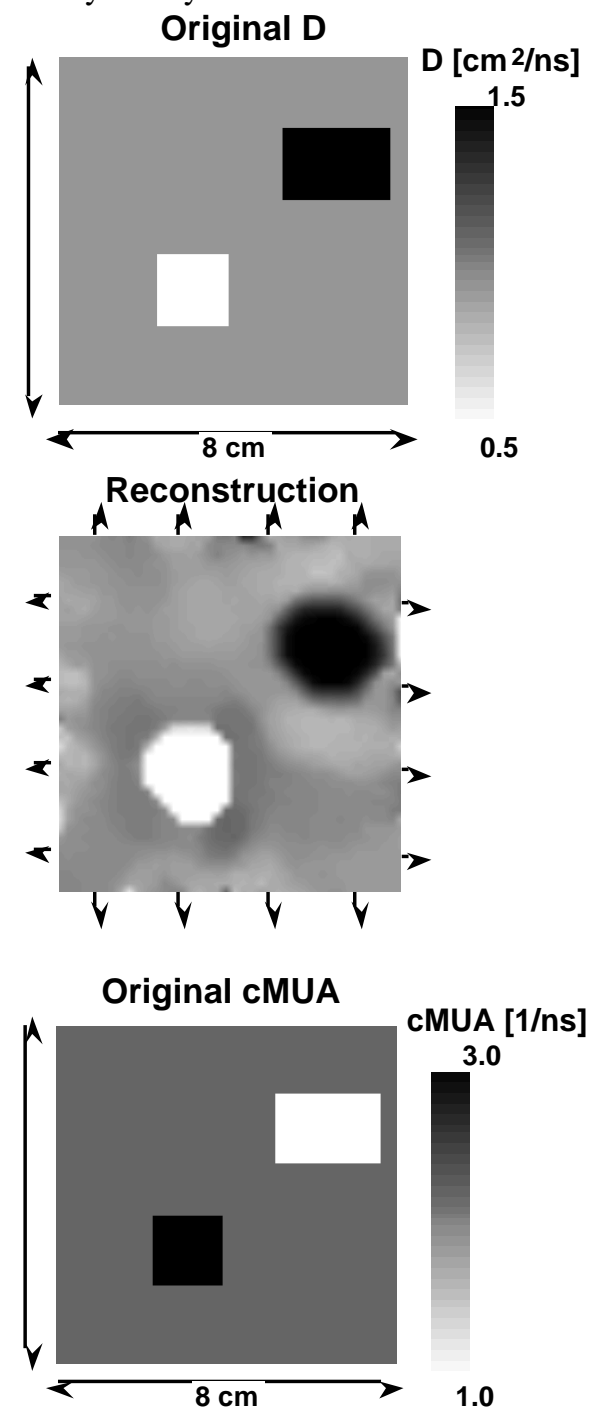




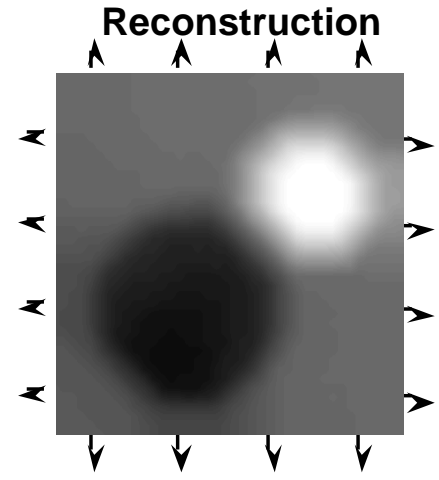

Fig. 2a,b (a-top) The original simple heterogeneous medium, which contains two inhomogeneities. The numbers in the figure represent the diffusion coefficient $D$, in $\mathrm{cm}^{2} \mathrm{~ns}^{-}$ 1 , at various locations in the media. (b-bottom) Here the reconstructed medium after 15 iterations (20 minutes) is shown. The arrows indicate the 16 source/detector positions surrounding the medium. In this case the SNR = $30 \mathrm{db}$.

\subsection{Brain Imaging}

To test the algorithms in a more realistic situation, MRI density maps were used to generated optical property maps of the brain. MRI imaging techniques allow to distinguish between skin, skull, white matter, gray matter, blood and cerebrospinal-fluid-filled spaces in the head. These different tissues appear in MRI scans with different densities. From this an optical property map $\left(\mathrm{c}_{\mathrm{a}}(\mathbf{r}), \mathrm{D}(\mathbf{r})\right)$ is obtained by assigning different optical properties to different density values. Fig. 3a shows such a segmented scan for a slice through the head with the variations in $D$ Outstanding features are the cerebro-spinal-fluid-filled ventricle in the center of the brain and a hematoma near the forehead. Furthermore, the brain is surrounded by cerebrospinal fluid as can be seen in the light areas in Fig. 3a. Figure $3 \mathrm{~b}$ shows the reconstruction based on measurements from 12 source-detector positions. In this case 60 iterations were performed which took about 70 min. While fine structures are not resolved, clearly visible in the reconstruction are the ventricles and other areas filled with cerebrospinal fluid. Also the hematoma at the forehead can be seen.

Figure $3 \mathrm{c}$ shows the same reconstruction, only this time the ventricles in the center of the brain were filled with blood. Ventricular bleeding often occurs in preterm infants and leads to server brain damage or death of the infant. The reconstructions shown in $3 \mathrm{~b}$ and c, clearly show that photon migration imaging can detect ventricular bleeding and can possibly be used as an early warning system, which allows physicians to react in time.

\subsection{Breast Imaging}

Another field of possible application of photon migration tomography is breast cancer detection. To simulate breast image reconstructions, an MRI-density map of the breast was converted into a map of optical properties. Fig. 4 shows such a segmented scan for a slice that contains the nipple and chestwall. Tumors consist of dense tissues that are highly vascularized, therefore $\mathrm{D}$ is lower. On the otherhand, cysts are filled with fluids that scatter less than the surrounding tissue. Therefore, D is higher. It was assumed that the breast is immersed in a matching fluid with optical properties similar to the one of the breast. This is a technique currently proposed by several researchers in the field.

The challenge in this type of problem is to detect the diseased tissue (here tumor and cysts) in a healthy heterogeneous background. For this example the spatial resolution was chosen to $\Delta \mathrm{x}=\Delta \mathrm{y}=0.28 \mathrm{~cm}$, resulting in a $32 \times 56$ grid for the finite-differencing scheme. 15 source/detector positions were assigned on three accessible sides of the breast.

In this example we used as initial guess and regularization term the information about the healthy breast (Fig. 8a; see also Eq. 9). Fig. 8b shows the result of the reconstruction after 10 iteration $(\sim 15 \mathrm{~min})$. The tumor and one cyst are clearly visible. Fig. $8 \mathrm{c}$ shows the ratio of the reconstructed image and the initial guess. The tumor

\section{Original}

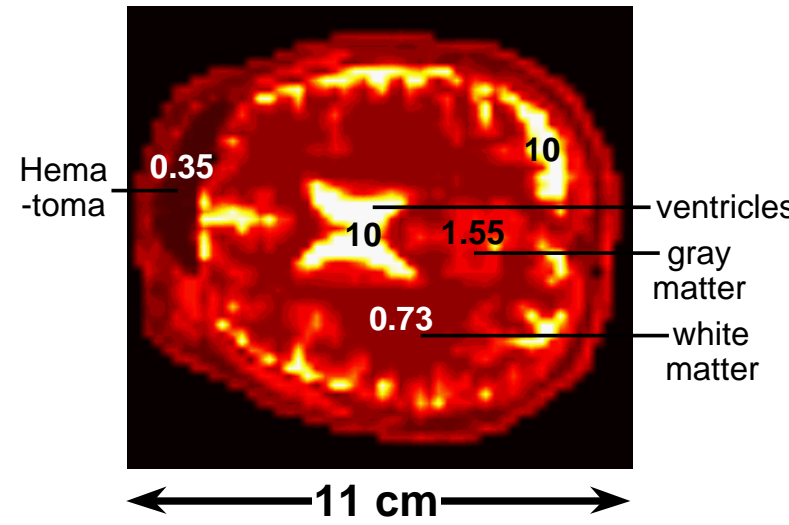

Reconstruction

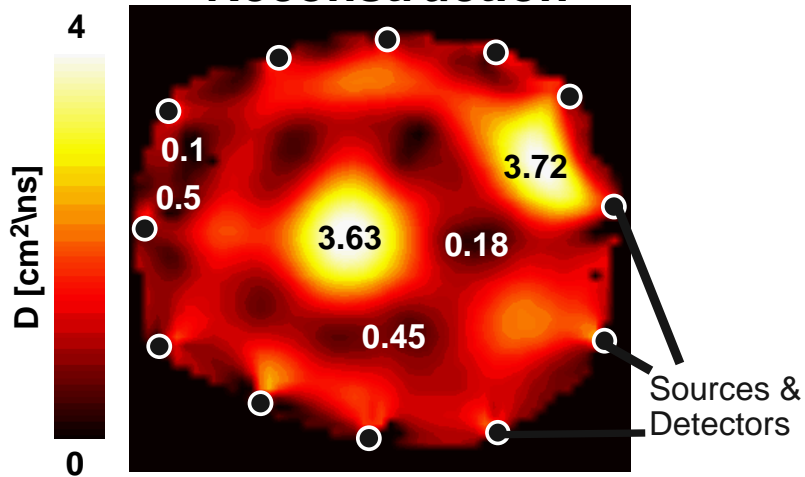




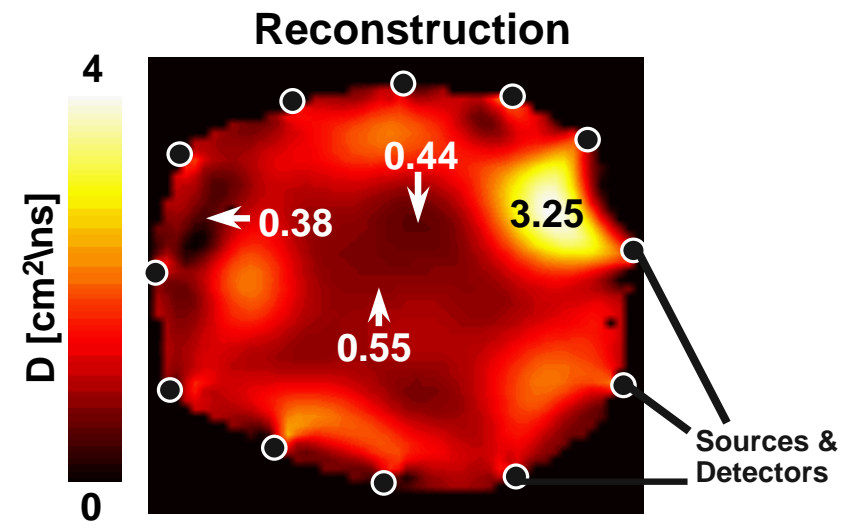

Fig. 3a,b,c: Reconstruction example of the brain. The numbers indicate the diffusion coefficient $D\left[\mathrm{~cm}^{2} \mathrm{~ns}^{-2}\right]$ at various positions. The spatial resolution is $0.2 \mathrm{~cm}$. The circles in the bottom image indicate source and detector positions.

and cyst becomes even more visible in this case. This type of procedure may be useful in cases where MRI data is available, and one needs to obtain additional information about a suspicious mass in the breast. Certain tumors or cyst may not show up on an MRI image but may be seen with optical techniques. The MRI-image could be used as an initial guess and the reconstruction would show optical irregularities.

(1)

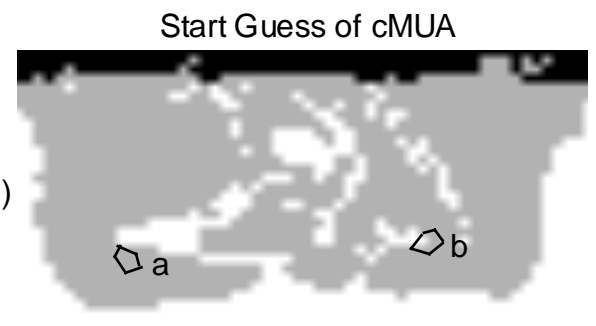
CMUA $[1 / \mathrm{ns}]$

(2)

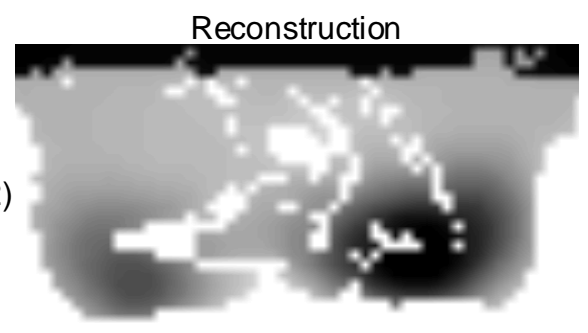

(Recon./Guess) of cMUA • (Recon./Guess) of D

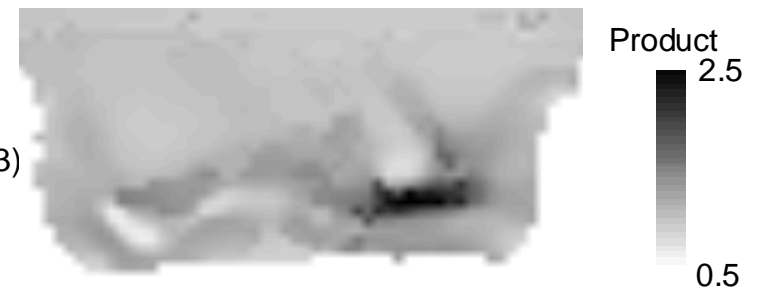

Fig. 4a,b,c: Image reconstruction of breast shown in Fig. 4a. In this case the initial guess is given by the healthy breast (a-top), which does not contain any tumors or cysts. The reconstruction after 30 iteration is shown in Fig. $4 b$ (middle). As can be seen in Fig. 4c(bottom), an even better localization is obtained by performing the product of the ratios of the reconstruction and the initial guess for $D$ and $c M U A$.

\section{Summary}

We have developed a model-based iterative reconstruction algorithm for time-resolved photon migration tomography. This algorithm does not require small perturbation or advance knowledge of a reference medium. The algorithm consists of three components: (1) a forward model that is used to predict the measurements assuming a certain distribution of optical properties. The forward model is currently based on a finite-difference formulation of the time-resolved diffusion equation; (2) an analysis scheme in which an objective function is defined, which needs to be minimized; (3) an updating scheme which allows to subsequent guess the optical properties of the medium based on the gradient of the objective function.

The code was successfully used to reconstruct simple heterogeneous media, which contain well defined object in a homogeneous background. The code was also tested on MRI generated optical property maps of the brain and the breast. The initial results are encouraging as tumors, cysts, and other inhomogeneities appear to be reconstructable even in an highly heterogeneous background.

\section{Acknowledgments}

This work was in part supported by a fellowship from the director's office at Los Alamos National Laboratory.

\section{References}

${ }^{1}$ B. Chance and R. R. Alfano, eds., Optical Tomography and Spectroscopy of Tissue: Theory, Instrumentation, Model, and Human Studies, Proc. 2979, SPIE-The International Society for Optical Engineering, Bellingham, WA 1997.

${ }^{2}$ R.R. Alfano and J.G. Fujimoto, eds., Advances in Optical Imaging and Photon Migrations, OSA Trends in Optics and Photonics Series, vol. 2, Optical Society of America, Washington, DC, 1996.

${ }^{3}$ B. Chance and R. R. Alfano, eds., Optical Tomography, Photon Migration and Spectroscopy of Tissue and Model Media: Theory, Human Studies, and Instrumentation, Proc. 2389, Part 1 and 2, SPIEThe Internat. Society for Optical Engineering, Bellingham, WA 1995.

${ }^{4}$ A. Yodh , B. Chance, "Spectroscopy and Imaging with diffusing light," Physics Today $\underline{48}, 34-40$ (1995).

${ }^{5}$ H. Jess, H. Erdl, K.T. Moesta, S. Fantini, M.A. Franceschini, E. Gratton, and M. Kaschke, " Intensity Modulated Breast Imaging: Technology and Clinical Pilot Study Results," OSA Trends in Optics and Photonics on Advances in Optical Imaging and Photon Migration, Vol. 2, R.R. Alfano and J.G. Fujimoto, eds., Optical Society of America, Washington, DC, pp. 126-129 (1996).

${ }^{6}$ J.P. Vanhouten, D.A. Benaron, S. Spilman, and D.K. Stevenson, "Imaging brain injury using time-resolved near-infrared light scanning," Pediatric Research 39, pp. 470-476, 1996. 
${ }^{7}$ S.P. Gopinath, C.S. Robertson, C.F. Contant, R.K. Narayan, R.G. Grossman, and B. Chance, "Early detection of delayed traumatic intracranial hematomas using near-infrared spectroscopy," J. Neurosurgery 83, 438-444 (1995).

${ }^{8}$ J. R. Mourant, T. M. Johnson, and D. S. Jack, "Physiological monitoring of brain function with a broadband, multifiber, continous-wave optical system," in Biomedical Sensing, Imaging, and Trackin Technologies II, Proc. SPIE 2976, Bellingham, WA, 1997, pp. 32-39.

${ }^{9}$ G. Gratton, M. Fabiani, D. Friedman, M.A. Franceschini, S. Fantini, P. Cobrallis, and E. Gratton, "Rapid changes of optical parameters in the human brain during a tapping task," J. Cognitive Neuroscience $\underline{7}$, 446456 (1995) . ????????

${ }^{10}$ K. Wells, J.C. Hebden. F.E.W. Schmidt, and D.T. Delpy, " The UCL multichannel time-resolved system for optical tomography", in Optical Tomograpgy and Spectroscopy of Tissue, B. Chance and R.R. Alfano, eds., Proc. SPIE 2979, in press. ??????

${ }^{11} \mathrm{M}$. Miwa and Y. Ueda, "Development of time-resolved spectroscopy system for quantitative noninvasive tissue measurement," in Optical Tomography, Photon Migration, and Spectroscopy of Tissue and Model Media, B. Chance and R.R. Alfano, eds., Proc. SPIE 2389, pp.142-149, 1995.

${ }^{12}$ S. Fantini, M.A. Franceschini, J.S. Maier, S.A. Walker, B. Barbieri, and E. Gratton, "Frequency domain multichannel optical detector for noninvasive tissue spectroscopy and oximetry," Opt. Eng. 34, 32-42 (1995).

${ }^{13}$ S.A. Walker, S. Fantini, and E. Gratton, "Image recon-struction by backprojection from frequency domain optical measurements in highly scattering media," Appl. Opt. $\underline{36}$, 170-179 (1997).

${ }^{14}$ S. B. Colak, D. G. Papaioannou, G. W. 't Hooft, M. B. van der Mark, H. Schomberg, J. C. J. Paasschens, J. B. M. Melissen, and N. A. A. J. van Asten, "Tomographic image reconstruction from optical projections in light-diffusing media," Appl. Opt. 36, 180-213 (1997).

${ }^{15}$ Y. Q. Yao, Y. Wang, Y. L. Pei, W. W. Zhu, R. L. Barbour, " Frequency-domain optical imaging of absorption and scattering distrinbutions by Born iterative method," J. Opt. Soc. Am. A 14, 325342 (1997)

${ }^{16}$ R. L. Barbour, H. L. Graber, J. W. Chang, S. L. S. Barbour, P. C. Koo, R. Aronson, "MRI-guided optical tomography: Prospects and computation for a new imaging method," IEEE Computational Science \& Engineering 2, 63-77 (1995).

${ }^{17}$ M. A. Oleary, D. A. Boas, A. G. Yodh, "Experimental images of heterogeneous turbid media by frequency-domain diffusing photon tomography," Opt. Lett. 20, 426-428 (1995).

${ }^{18}$ M. Schweiger and S.R. Arridge, " A system for solving the forward and Inverse Problems in optical spectroscopy and imaging," in Advances in Optical Imaging and Photon Migrations, OSA Trends in Optics and Photonics Series, vol. 2, R.R. Alfano and J.G. Fujimoto, eds., Optical Society of America, Washington, DC, 1996.

${ }^{19}$ H. B. Jiang, K. D. Paulsen, U. L. Osterberg, B. W. Pogue, and M. S. Patterson, "Optical image recon-struction using frequency domain data: Simulations and experiments," J. Opt. Soc. Am. A $\underline{13}$, 253-266 (1996).

${ }^{20}$ S. S. Saquib, K. M. Hanson, and G. S. Cunningham, "Model-based image reconstruction from time-resolved diffusion data", in Medical Imaging: Image Processing, Proc. SPIE 3034, pp. 369-380, 1997.

${ }^{21}$ A.H. Hielscher, "Model-Based Iterative Image Recon-struction for Photon Migration Tomography," in Methods for Solving Ill-Posed Inverse Imaging Problems: Medical and Nonmedical Applications, R.L. Barbour, M.J. Carvlin, and M.A. Fiddy, eds., SPIE- Proc. 3171, pp.106-117, 1997.

${ }^{22}$ W.H. Press, S. A. Teukolsky, W.T. Vetterling, and B.P. Flannery, Numerical Recipes in C, Cambridge University Press, New York, NY, 1992.

${ }^{23}$ G. Birkhoff and R.E. Lynch, Numerical Solution of Elliptical Problems, SIAM, Philadelphia, PA, 1984, pp. 173-178.
${ }^{24}$ W.F. Ames, Numerical Methods for Partial Differential Equations, Academic Press, New York, NY, 1977, pp. 148-157.

${ }^{25}$ D.V. O'Connor and D. Phillips, Time-correlated single photon counting, Academic Press, Orlando, FL, 1984.

${ }^{26}$ H. Liu, M. Miwa, B. Beauvoit, N.G. Wang,a nd B. Chance, "Characterization of absorption and scatetring properties of small volume biological samples using time-resolved spectroscopy, Anal. Biochem. 213, 378-385 (1993). 